\title{
Tyrosine phosphorylation of PP2A is regulated by HER-2 signalling and correlates with breast cancer progression
}

\author{
LEE LEE WONG ${ }^{1}$, CHAN FONG CHANG $^{2}$, EVELYN S.C. KOAY ${ }^{1,3}$ and DAOHAI ZHANG ${ }^{1,3}$ \\ Departments of ${ }^{1}$ Pathology and ${ }^{2}$ Biochemistry, Yong Loo Lin School of Medicine, National University \\ of Singapore; ${ }^{3}$ Department of Laboratory Medicine, National University Hospital, \\ National University Health System, Singapore 119074
}

Received December 15, 2008; Accepted February 11, 2009

DOI: 10.3892/ijo_00000256

\begin{abstract}
Activation of HER-2/neu leads to multiple signalling cascades and plays a vital role in cell survival and growth. We used a signal transduction antibody array to characterize the tyrosine phosphorylation profiles in heregulin (HRG $\alpha 1$ )-treated BT474 breast cancer cells, and identified a group of 80 molecules in which tyrosine phosphorylation was highly regulated by HRG-enhanced HER-2/ neu signalling. These phosphoproteins included many known HER-2/neu-regulated molecules (e.g., SHC, Akt, Syk and Stat1) and proteins that had not been previously linked to HER-2/neu signalling, such as Fas-associated death domain protein (FADD), apoptosis repressor with CARD domain (ARC), and the tumour suppressor, protein phosphatase type 2A (PP2A). Pharmacological inhibition with HER-2 inhibitor AG825, PI3K inhibitor LY294002, MEK1/2 inhibitor PD98095, and p38MAPK inhibitor SB203580 confirmed that PP2A phosphorylation was modulated by the
\end{abstract}

Correspondence to: Dr Daohai Zhang, Department of Laboratory Medicine, National University Hospital, National University Health System, 5 Lower Kent Ridge Rd, Singapore 119074

E-mail: daohai_zhang@nuhs.edu.sg

Dr E.S.C. Koay, Department of Pathology, YLL School of Medicine, NUS, National University Health System, NUH Main Building, Level 3, 5 Lower Kent Ridge Rd, Singapore 119074

E-mail: patkoaye@nus.edu.sg

Abbreviations: HER-2/neu, human tyrosine kinase-type cell surface receptor, type 2; EGFR, epidermal growth factor receptor; HRG, heregulin $\alpha 1$; PI3K, phosphatidylinositol-3 kinase; MAPK, mitogen-activated protein kinase; $\mathrm{PP} 2 \mathrm{~A} / \mathrm{C}$, protein phosphatase type $2 \mathrm{~A}$ catalytic subunit; AKT, v-akt murine thymoma viral oncogene homolog; MEK1/2, mitogen-activated protein kinase 1/2; FADD, Fas-associated death domain protein; ARC, apoptosis repressor with CARD domain; p38MAPK, p38 mitogen-activated protein kinase; ERK, extracellular signal-regulated kinase; $\mathrm{p}-\mathrm{Y}$, phosphotyrosine; DMSO, dimethyl sulfoxide; HRP, horseradish peroxidase; Raf, v-raf-1 murine leukemia viral oncogene homolog 1

Key words: HER-2/neu, protein phosphatase 2A, tyrosine phosphorylation, $\mathrm{PI} 3 \mathrm{~K} / \mathrm{p} 38 \mathrm{MAPK}$, breast cancer complicated, HER-2/neu-driven downstream signal network, with the PI3K and MEK1/2 positively, while the p38MAPK negatively regulating its tyrosine phosphorylation. In breast tumour specimens, expression of tyrosine-phosphorylated PP2A (pY307-PP2A) was highly increased in the HER-2/neu positive breast tumours, and significantly correlated to tumour progression, thus enhancing its potential prognostic value. Our data provide meaningful information in the elucidation of the HER-2-driven tyrosine phosphorylation network, and in the development of phosphopeptide-related targets as prognostication indicators.

\section{Introduction}

ErbB2, the HER-2/neu-encoded transmembrane protein, is a member of the family of receptor tyrosine kinases (RTKs) (1). The RTKs command central roles in several signal transduction pathways regulating cell proliferation, differentiation, and survival (2). Constitutive activation of HER-2 occurs in $20-30 \%$ of breast cancers and is associated with poorer prognosis and a more aggressive phenotype (3). Clinically, targeting HER-2 with a monoclonal antibody, Herceptin, to inhibit its kinase activity is an effective supplementary therapy for HER-2 positive breast cancer patients. However, relapse or resistance to this chemotherapy has frequently occurred and alternative treatment regimes have to be developed (4). A full understanding of the contribution of HER-2 signalling to oncogenesis is a critical step in the search for novel and more effective therapeutic targets for breast cancer.

HER-2/neu is a ligandless receptor with an extracellular domain, a transmembrane domain and a C-terminal intracellular domain. Receptor dimerization of HER-2 with other RTK members induces the activation of its downstream signalling by autophosphorylation of tyrosine residues at its C-terminal sites and recruitment of other docking molecules, such as Grb2, Stat and Shc. The key HER-2-regulated signalling pathways include the Ras/Raf/mitogen-activated protein kinase (MAPK) and phosatidylinositol-3 kinase (PI3K) pathways, which are essential for cell proliferation and transformation (5). However, the detailed signalling network regulated by HER-2 signalling is largely unclear. Dissecting the complexity of protein phosphorylation and dephosphorylation events in tumour cells remains an area of intense research. 
Recently, a group of tyrosine-phosphorylated proteins regulated by HER-2 signalling have been identified using proteomics strategies (6-10). By profiling the tyrosinephosphorylated proteins in ErbB2-overexpressing breast and ovarian cancer cell lines, Mukherji et al found that the adaptor/docking proteins, kinases and other proteins involved in cell proliferation and migration were highly tyrosinephosphorylated and treatment with Herceptin attenuated their phosphorylation (6). In addition, several uncharacterized RNA binding proteins, e.g., SRRM2 and SFRS9, were also highly phosphorylated in the HER-2-overexpressed cells (6). When these cells were treated with Heregulin $\alpha 1$ (HRG), an EGF-like growth factor, proteins such as paxillin, FAK and GIT1 were highly tyrosine-phosphorylated and the signal network involved in cell migration was amplified (9). Bose et al reported that inhibition of HER-2 signalling with the specific tyrosine kinase inhibitor, PD168393, led to the identification of several previously uncharacterized phosphoproteins, including Axl tyrosine kinase, the adaptor protein Fyb and the calcium-binding protein PDCD-6/Alg-2 (7). It is possible, with appropriate use of different complementary phosphoproteomics investigative methods, to dissect, identify and characterize the tyrosine-phosphorylated proteins in the HER-2-driven signal network and achieve a better understanding of the mechanisms underlying the cellular response. Profiling the tyrosine-phosphorylated proteome is the logical first step to elucidate the dynamic phosphorylation and dephosphorylation of a variety of proteins which constitute the complicated network controlling cellular behaviour under the influence of receptor tyrosine kinase signaling.

To further understand the complexity of tyrosinephosphorylation profiles of the interactive downstream proteins of the HER-2 signalling, we treated the ErbB2expressing BT474 cells with HRG and investigated the differential tyrosine phosphorylation of various signal modulators using an antibody array. We identified a group of signal modulators which are differentially tyrosinephosphorylated by HRG-enhanced HER-2 signalling. Postmodification of these signal modulators are diversely involved in multiple cellular functions, primarily in cell apoptosis, signal transduction, transcription, cell cycle regulation, and cell proliferation. In particular, we investigated the role of HER-2 signalling in modulating tyrosine phosphorylation of protein phosphatase 2A (PP2A), one of the identified signal modulators. Inhibition studies with various inhibitors showed that the PI3K/Akt and Raf/Ras/MEK pathways positively regulate, whereas the p38MAPK pathway negatively regulates the tyrosine phosphorylation of PP2A. Furthermore, as the level of tyrosine-phosphorylated PP2A at tyrosine 307 (pY307-PP2A) was found to be increased in the HER-2-positive breast cancer subgroup, the clinical significance of this as a prognostication biomarker of cancer progression was further assessed in a panel of 206 clinical specimens.

\section{Materials and methods}

Materials. LY294002, a specific inhibitor of the p110 catalytic subunit of PI3K, AG825, a specific inhibitor of HER-2/neu tyrosine kinase, and SB203580, a specific inhibitor of p38MAPK, were purchased from Calbiochem (San Diego, CA, USA). PD98059, a specific inhibitor of MEK, was from US Biological (Swampscott, MA, USA). Heregulin $\alpha 1$ (HRG), a ligand of ErbB3 and HER-2/neu (ErbB2), was from Neomarkers (Fremont, CA). All the inhibitors were dissolved in dimethyl sulfoxide (DMSO) and stored at $-20^{\circ} \mathrm{C}$ in the dark as stock solutions until use.

The following antibodies were used for immunoblot analysis: rabbit anti-phospho-HER-2/neu (Tyr1248) (1:1000), rabbit anti-ERK1/2 (1:1000), mouse anti-phosphoERK1/2 (Tyr204) (1:500), rabbit anti-ARC (1:200), rabbit anti-FADD (1:200), rabbit anti-N-Shc (1:1000), rabbit antiphospho-Shc (Tyr239/240) (1:200), rabbit anti-PP2A (1:200), rabbit anti-phospho-PP2A (Tyr307) (1:1000), mouse anti-Stat1 (1:200), and rabbit anti-phospho-Stat1 (Tyr701) (1:200), were obtained from Santa Cruz Biotechnology (Santa Cruz, CA, USA). Mouse anti-phosphotyrosine 4G10 (1:1000) was purchased from Upstate Biotechnology Inc. (Lake Placid, NY), mouse anti-HER-2/neu (1:1000) from Neomarkers, mouse anti-Ezrin (1:100) from BD Biosciences PharMingen (San Diego, CA, USA) and horseradish peroxidase (HRP)-conjugated phosphotyrosine (1:1000) from Hypromatrix (Worcester, MA, USA). Rabbit anti-AKT (1:1000) and rabbit anti-phospho-AKT (Ser473) (1:1000) were obtained from Cell Signalling Technology (Beverley, MD, USA).

Cell culture and treatment. The human HER-2/neu positive BT474 breast cancer cell line was grown in Dulbecco's modified Eagle's medium (DMEM) supplemented with $10 \%$ fetal bovine serum (FBS) at $37^{\circ} \mathrm{C}$ in a humidified $5 \% \mathrm{CO}_{2}$ incubator. For HRG stimulation, BT474 cells (70-80\% confluence) were serum-starved for $20 \mathrm{~h}$ and $\mathrm{HRG}$ (final concentration $3 \mathrm{nM}$ ) was added into the medium. Cells were then incubated for the indicated time periods $(5,10$ and $30 \mathrm{~min}$ ). For the inhibitor treatment (with AG825, SB203580, PD98059 and LY294002), the BT474 cells (at 70-80\% confluence) were fed with fresh medium, supplemented with different individual inhibitors at the indicated concentrations (as stated in the context) for 10 and $30 \mathrm{~min}$. Cells were then harvested for Western blotting. Cells treated with an equal volume of DMSO were used as control. Each treatment was performed in triplicates and each treatment was repeated thrice.

To prepare cell lysates, BT474 cells were collected after trypsin-treatment and washed 3 times with ice-cold phosphate-buffered saline (PBS, pH 7.4). Cells were resuspended in cold RIPA buffer (1\% Igepal, $1 \%$ sodium deoxycholate, $0.15 \mathrm{M}$ sodium chloride, $0.01 \mathrm{M}$ sodium phosphate, $\mathrm{pH} 7.2$, and $2 \mathrm{mM}$ EDTA) supplemented with protease inhibitors (Roche Diagnostics, Indianapolis, IN, USA) and phosphatase cocktail inhibitors I and II (1:100; Sigma-Aldrich, Steinheim, Germany), and kept on ice for $30 \mathrm{~min}$. Cell lysates were then centrifuged at $4{ }^{\circ} \mathrm{C}$ and the supernatants were collected. Protein concentrations were determined using the Coomassie Plus Bradford assay (Pierce, Rockford, IL, USA).

Identification of tyrosine-phosphorylated proteins using signal transduction antibody array. The serum-starved BT474 cells were treated with HRG (3 nM) or with DMSO for $30 \mathrm{~min}$ and the total proteins were extracted. After the 
array was blocked with $5 \%$ non-fat milk in Tris-buffered saline with $0.1 \%$ Tween-20 (TBST) for $1 \mathrm{~h}$, equal amounts of proteins from both the HRG-treated and the DMSO-treated (control) cells were diluted to $1 \mathrm{mg} / \mathrm{ml}$ and then probed with the Signal Transduction Antibody Array (Hypromatrix), respectively, for $2 \mathrm{~h}$ at room temperature, according to the manufacturer's instructions. The arrays were washed with TBST buffer and further incubated with HRP-conjugated anti-phosphotyrosine antibody (Hypromatrix) for $2 \mathrm{~h}$ with gentle agitation. The chemiluminescent signals were then visualized using the SuperSignal ${ }^{\circledR}$ West Pico Chemiluminescent Substrate (Pierce) and captured with the MULTI GENIUS Bio Imaging System (Syngene, Frederick, MD, USA). The signal intensity of each spot was analyzed using the GeneTools software (Syngene). The relative tyrosine phosphorylation level of each protein was interpreted as the ratio of the spot intensity in the HRG-treated sample to the spot intensity in the control sample.

Immunoprecipitation and Western blotting. For immunoprecipitation, $500 \mu \mathrm{g}$ of proteins was immunoprecipitated with selective antibodies (anti-FADD, anti-ARC, and antiEzrin) at $4^{\circ} \mathrm{C}$ and gently agitated overnight. Protein ASepharose or protein G-Sepharose (GE Healthcare, Uppsala, Sweden) was then added and the mixture incubated at $4{ }^{\circ} \mathrm{C}$ for $2 \mathrm{~h}$. The agarose beads were collected, washed and resuspended in $50 \mu \mathrm{l}$ of sample buffer containing $50 \mathrm{mM}$ Tris-HCl, pH 7.6, 2\% SDS, 10\% glycerol, $10 \mathrm{mM}$ DTT and $0.2 \%$ bromophenol blue. After boiling for $5 \mathrm{~min}$, samples were cooled down on ice and analyzed by Western blotting. Tyrosine phosphorylation was detected using anti-phosphotyrosine antibody. Briefly, proteins were separated by SDSPAGE and then transferred to Hybond-P PVDF membrane (GE Healthcare). The filters were blocked in 5\% non-fat milk in TBST for $1 \mathrm{~h}$ at room temperature and probed with the respective antibodies at $4^{\circ} \mathrm{C}$ overnight. HRP-conjugated goat anti-mouse $\operatorname{IgG}$ (1:5000, Upstate) or HRP-conjugated goat anti-rabbit IgG (1:10000, ZyMED Laboratories Inc., San Francisco, CA) was applied after the filters were washed with TBST. The chemiluminescent signals were then detected and captured, as stated in the previous section.

Two-dimensional electrophoresis and immunoblotting. Protein separation by 2-dimensional electrophoresis (2-DE) was carried out as previously described (11). Briefly, $50 \mu \mathrm{g}$ of protein was mixed with $125 \mu 1$ of rehydration buffer $(7 \mathrm{M}$ urea, 2\% CHAPS, $3.5 \mathrm{mg}$ DTT and 1\% IPG buffer, pH 3.0-10.0 or $\mathrm{pH} 4.0-7.0)$ and separated using precast Immobiline IPG DryStrip (70 mm, pH 3.0-10.0 or pH 4.0-7.0, GE Healthcare) with the Ettan ${ }^{\mathrm{TM}}$ IPGphor ${ }^{\mathrm{TM}}$ Isoelectric Focusing System (GE Healthcare). Prior to SDS-PAGE, the strips were then equilibrated for $20 \mathrm{~min}$ in equilibration buffer $(50 \mathrm{mM}$ Tris- $\mathrm{HCl}$, $\mathrm{pH} 8.8,30 \%$ glycerol, $7 \mathrm{M}$ urea, 2\% SDS) containing DTT (100 mg per strip), followed by further equilibration with the same buffer with iodoacetamide (250 mg per strip). Protein transfer and immunoblotting were performed and signals were detected, as described above.

Immunohistochemistry and tissue microarray. A breast disease spectrum tissue array including 206 cases was

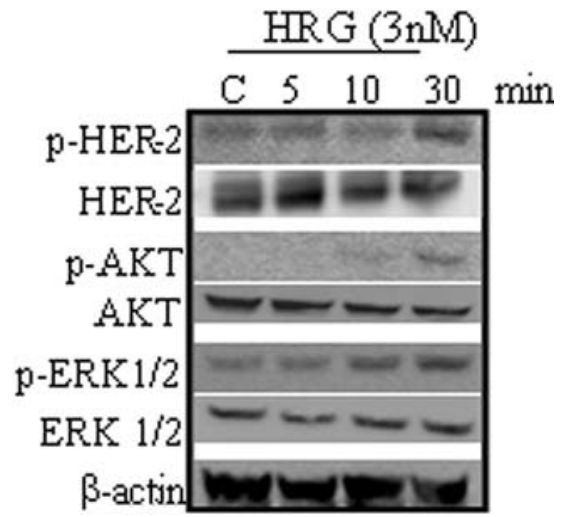

Figure 1. Stimulation of HRG $\alpha 1$ on HER-2 phosphorylation and its downstream signalling. BT474 breast cancer cells at 70-80\% confluence were serum-starved for $20 \mathrm{~h}$ and then treated with HRG $\alpha 1(3 \mathrm{nM})$ or DMSO (control) for 5, 10 and $30 \mathrm{~min}$. Cells were harvested and phosphorylation of HER2, AKT and ERK1/2 were determined by Western blotting with their respective antibodies as described in Materials and methods. The relative phosphorylation levels of HER2, ERK1/2 and AKT were normalized to their respective basal levels. Significant difference of treatment with control at different time intervals was analyzed using the Student's t-test. ${ }^{*} \mathrm{p}<0.05$, ${ }^{* *} \mathrm{p}<0.01$. Data represent average of triplicate experiments.

purchased from US Biomax (Rockville, MD, USA). The array contained 32 metastatic carcinomas, 68 invasive ductal carcinomas, 22 each of lobular and intraductal carcinomas, 16 normal tissues, and 44 other non-malignant tissues. Immunohistochemical (IHC) staining of pY307-PP2A was conducted using the Dako Envision system (11). Briefly, sections were deparaffinized, rehydrated, and the antigens unmasked using the Dako ${ }^{\circledR}$ Target Retrieval Solution. Endogenous peroxidases were removed using 3\% hydrogen peroxide, followed by incubating with anti-pY307-PP2A (1:25) antibody for $1 \mathrm{~h}$ at room temperature. The sections were further treated with labelled dextran polymer conjugated with HRP for $30 \mathrm{~min}$ and incubated with $\mathrm{DAB}^{+-}$ substrate chromogen solution for 5-10 min. Sections were counterstained with Mayer's hematoxylin and mounted. The degree of staining of pY307-PP2A was scored as 0 (negative to weak), 1 (moderate) and 2 (strong), according to the staining intensity of the tumour cells. Scoring was performed by two pathologists and the principal investigator, independently. Cases with discrepant scores were re-scored by the same or additional scorers, to obtain a consensus score, failing which the scores were excluded from the data analysis.

Statistical analysis. The Student's t-test was used to compare the significance of differences of relative tyrosine phosphorylation of PP2A (pY307-PP2A/PP2A) between controls and treated samples. The difference of tyrosinephosphorylated PP2A (pY307-PP2A) between different types of tissues (lymph node metastasis, malignant, nonmalignant and normal tissues) was analyzed with Fisher's exact test. Two-sided $\mathrm{p}<0.05$ was considered as significant.

\section{Results}

Identification of tyrosine-phosphorylated molecules regulated by heregulin-enhanced HER-2 signalling. HRG $\alpha 1$, a ligand 


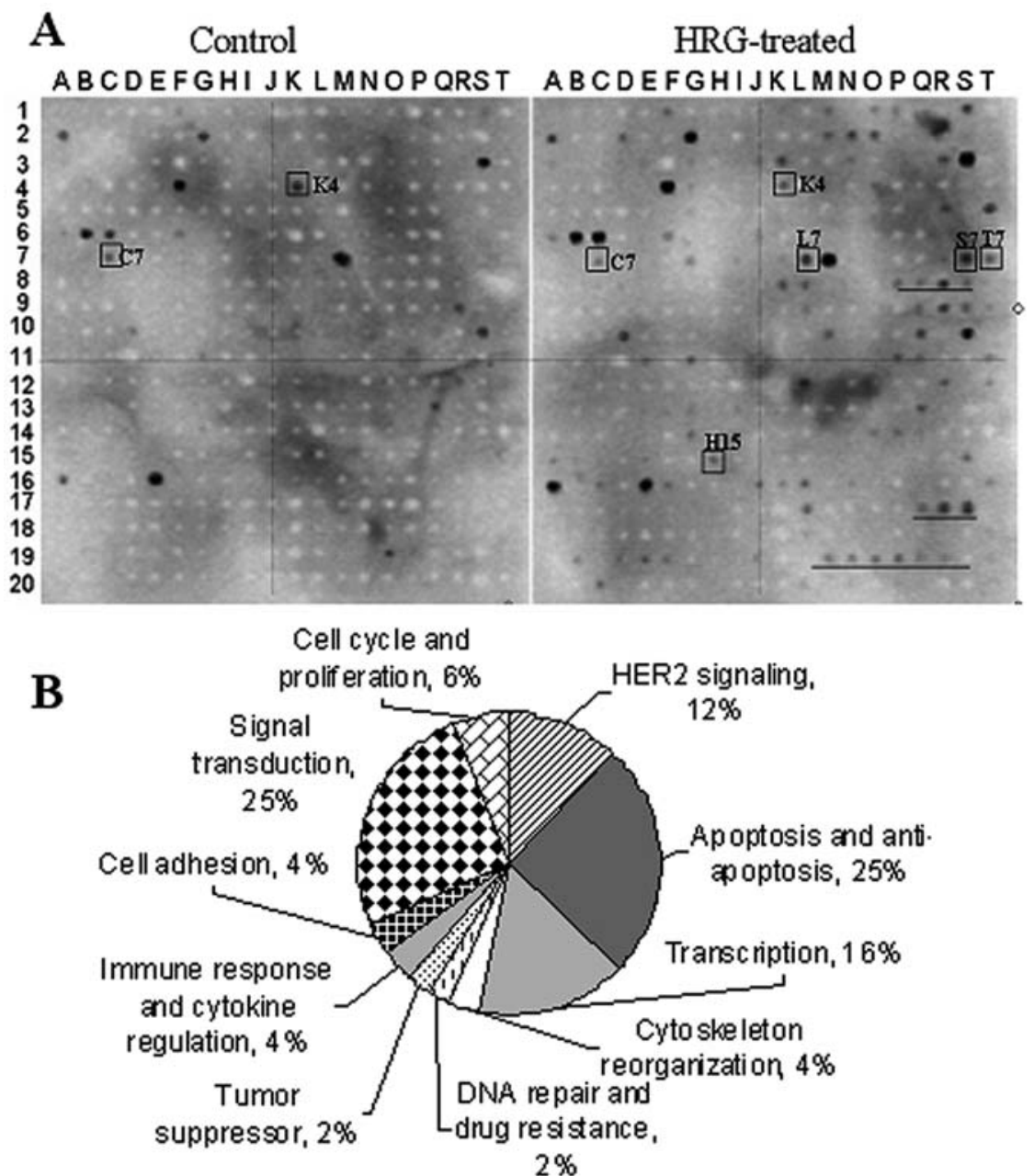

Figure 2. Differential tyrosine phosphorylation profiles on signal transduction antibody array between the HRG-treated and DMSO-treated (control) BT474 cells. (A) Representative filters showing the differential tyrosine phosphorylation profiles. Serum-starved BT474 cells (70-80\% confluence) were treated with HRG or DMSO for 30 min and equal amounts of proteins were separately incubated with two signal transduction arrays. An anti-phosphotyrosine antibody conjugated to horseradish peroxidase (HRP) was used to detect phosphorylated tyrosines on proteins. Signals were captured with the MULTI GENIUS Bio Imaging System (Syngene) and the signal intensity of each spot was analyzed using the Syngene GeneTools software. Relative tyrosine phosphorylation level of each protein was interpreted as the ratio of spot intensity in the HRG-treated sample to the spot intensity in the control sample. Spots K4 and C7 represent p-EGFR and CD3 epsilon with reduced phosphotyrosine signals in the HRG-treated BT474 cells. Other spots (L7: HER2; S7: Ezrin; T7: FADD and H15: PP2A) show the increased phosphotyrosine signals in the HRG-treated BT474 cells. Underlined spots represent the proteins involved in cell apoptosis. (B) Grouping of identified tyrosine phosphorylated and -dephosphorylated molecules. Only the proteins with a ratio of at least 1.5 -fold difference or only detected in either of the two filters were selected and functionally grouped in the pie chart.

of ErbB3, stimulates the heterodimerization of ErbB3 with ErbB2 and activates the downstream signalling (12). Treatment of the HER-2-expressing BT474 breast cancer cells with HRG showed the time-dependent phosphorylation of HER-2 and its key downstream modulators including AKT and ERK1/2 (Fig. 1). When the cells were treated for $30 \mathrm{~min}$, phosphorylation of ErbB2, ERK1/2 and AKT were highly enhanced, as determined by Western blotting with the respective phospho-specific antibodies. To investigate the tyrosine-phosphorylated molecules regulated by HER-2/neu signalling, the BT474 cells were treated with HRG or DMSO (as control) for $30 \mathrm{~min}$ and signal transduction antibody arrays were used to study the changes of tyrosine phosphorylation of different signalling modulators. Fig. 2A shows the differential tyrosine phosphorylation patterns on the filter between the HRG-treated and control BT474 cells. We found that 80 of the 400 arrayed molecules (20\%) showed significant differences in the spot intensities of treated samples vs. controls. The tyrosine-phosphorylated and dephosphorylated proteins identified are listed in Table I.

Functional analysis (Gene Ontology) showed that these proteins are mainly involved in cell apoptosis (25\%), signal transduction (25\%) and transcriptional activity (16\%) (Fig. 2B). Not surprisingly, we found several known HER-2/neuregulated proteins, e.g. Src homology 2 domain-containing transforming protein (SHC), Stat-1 transcription factor, Srcrelated protein tyrosine kinases (Syk), ErbB2, and AKT, are highly tyrosine-phosphorylated after HRG treatment, which further confirms the efficiency of the antibody array in dissecting the tyrosine-phosphorylated protein profiles. Most importantly, we also found a group of proteins whose tyrosine phosphorylations have not been previously linked to HER-2 signalling. For example, cell apoptosis-related proteins such as apoptosis repressor with CARD domain protein (ARC) and FAS-associated Death Domain protein (FADD), the cell motility-associated protein villin 2 (Ezrin), and protein 

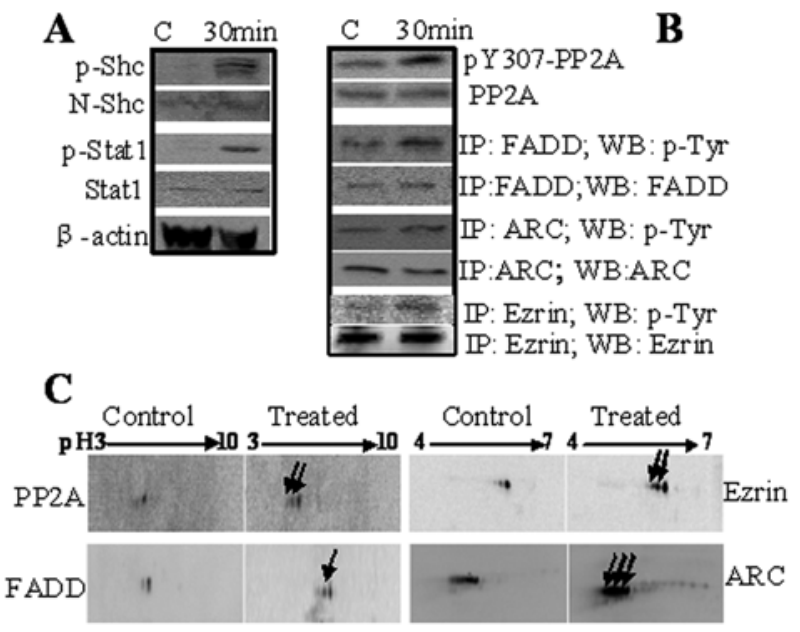

Figure 3. Validation of differential tyrosine phosphorylation of identified proteins by Western blotting. (A) Known proteins associated with HER2 signalling. (B) Novel tyrosine-phosphorylated proteins under HER2 signalling. BT474 breast cancer cells (70-80\% confluence) were treated with HRG $\alpha 1(3 \mathrm{nM})$ or DMSO for $30 \mathrm{~min}$, and equal amount of proteins were used for Western blotting, as described in Materials and methods. The levels of proteins and their tyrosine-phosphorylated forms were detected with their respective specific antibodies. For proteins (FADD, ARC and Ezrin) without specific phosphotyrosine antibodies, immunoprecipitation (IP) was carried out with the primary antibody and protein A-Sepharose or protein GSepharose. The protein complexes were separated and the tyrosine phosphorylation was detected with anti-phosphotyrosine antibody. (C) 2-dimensional electrophoresis and immunoblot. Equal amounts of proteins $(50 \mu \mathrm{g})$ from both HRG-treated and control BT474 cells were separated with IPG strips (70 mm, pH 3.0-10.0 or $\mathrm{pH} 4.0-7.0$ ), followed by SDS-PAGE. Protein spots were detected with their respective antibodies and visualized with chemiluminescent substrate as described in Materials and methods. Arrows indicated the enhancement of multi-spots in HRG-treat BT474 cells. IP, immunoprecipitation; WB, Western blotting.

phosphatase type 2A (PP2A), were highly tyrosine-phosphorylated in the HRG-treated BT474 cells. Interestingly, only 2 proteins, p-EGFR and CD3 epsilon (Fig. 2A, located at K4 and C7) were found to be highly dephosphorylated under the HRG-enhanced HER-2 signalling. Collectively, we identified a large number of HER-2-regulated tyrosinephosphorylated and -dephosphorylated signal proteins, including several novel signal modulators.

To confirm the HRG-enhanced tyrosine phosphorylation of the signal proteins identified by the antibody array, we treated the BT474 cells with HRG for $30 \mathrm{~min}$ and analysed the tyrosine phosphorylated levels of 2 proteins (Shc and Stat1) that are directly regulated by HER-2 signalling, and 4 other proteins (FADD, ARC, Ezrin and PP2A) that had not been previously reported to be associated with HER-2 signalling, by immunoprecitation and Western blotting. As shown in Fig. 3A, HRG significantly enhanced phosphorylation of Shc and Stat1, two important mediators in the HER-2 signalling pathway (13), relative to that observed in the control cells treated with DMSO. Similarly, tyrosine phosphorylation of PP2A, Ezrin, ARC and FADD were highly increased after HRG treatment (Fig. 3B). Immunoblot analysis of these 4 proteins after 2-DE separation revealed additional multi-spots with increased intensities, appearing on the acidic side of the main spots of PP2A, Ezrin, FADD and ARC in the HRG-treated cells (Fig. 3C), giving further

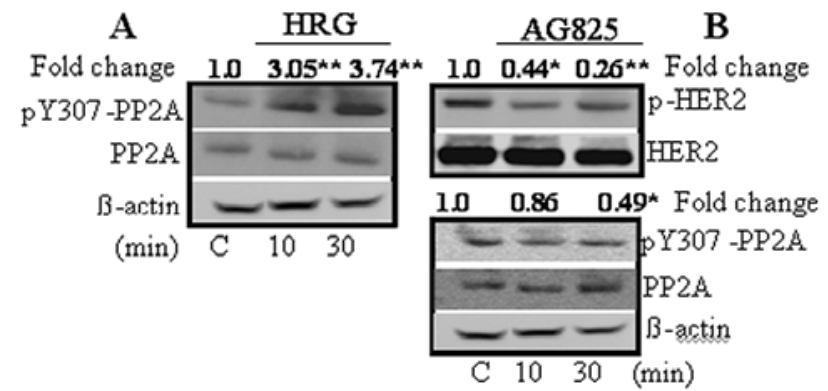

Figure 4. Regulation of tyrosine phosphorylation (pY307) of PP2A by HER2 signalling. (A) Time-dependent tyrosine phosphorylation of PP2A by HRG treatment. Serum-starved BT474 cells were treated with HRG for 10 and $30 \mathrm{~min}$ and the level of PP2A and its tyrosine-phosphorylated form pY307-PP2A were detected by Western blotting. (B) Reduction of pY307 of PP2A by HER2 kinase inhibitor AG825. BT474 cells at $70-80 \%$ confluence were treated with AG825 $(100 \mu \mathrm{M})$ for 10 and $30 \mathrm{~min}$ and the phosphoHER2, HER2, PP2A and pY307-PP2A were detected with their respective antibodies by Western blotting. Relative tyrosine phosphorylation level of HER2 and PP2A were normalized with its basal level (p-HER2/HER2, pY307-PP2A/PP2A), respectively. Fold change of relative phosphorylation was interpreted as the ratio when considering the relative phosphorylation level in control as 1. Data represent the average of triplicate experiments. The Student's t-test was used for the analysis of significance. ${ }^{*} \mathrm{p}<0.05$, ${ }^{* *} \mathrm{p}<0.01$.

evidences of phosphorylation modification of these proteins under HRG stimulation.

HER2 signalling regulates tyrosine phosphorylation of PP2A. PP2A is one of the major serine/threonine phosphatases involved in multi-cellular processes including regulation of different signal transduction pathways (e.g., Raf/MEK/ERK and PI3K/Akt) and control of cell cycle progression, gene transcription and protein translation (14-17). It was reported that PP2A could be inactivated by tyrosine phosphorylation in response to cell growth stimulation by EGFR or c-Src (18). In our study, we found that PP2A was highly tyrosinephosphorylated at tyrosine 307 (pY307) in a time-dependent manner when HER-2 signalling was enhanced in the HRGtreated BT474 breast cancer cells (Fig. 4A). To further verify the role of HER-2 signalling in the regulation of PP2A phosphorylation, we blocked the HER-2 signalling by treating BT474 cells with tyrphostin AG825, a specific HER-2 kinase inhibitor (19). Just as the phosphorylation of HER-2 was reduced in cells treated with AG825 $(100 \mu \mathrm{M})$, pY307 of PP2A was decreased in the same way (Fig. 4B). These results demonstrate the regulatory role of HER-2 signalling in tyrosine phosphorylation of PP2A.

PI3K/AKT and MEK/ERK positively regulate, whereas $p 38$ MAPK negatively regulates tyrosine phosphorylation of $P P 2 A$. HER-2 signalling activates several downstream signalling pathways, e.g., PI3K/Akt, thus leading to dysregulated cell proliferation and enhanced cell survival. To further understand the regulatory mechanism of PP $2 \mathrm{~A}$ phosphorylation driven by HER-2 signalling, different inhibitors targeting PI3K, MEK, and P38MAPK, were used to block the respective pathway and the level of phosphorylated PP2A at its tyrosine 307 residue (pY307-PP2A) was analyzed by Western blotting. As shown in Fig. 5A, when the 
A

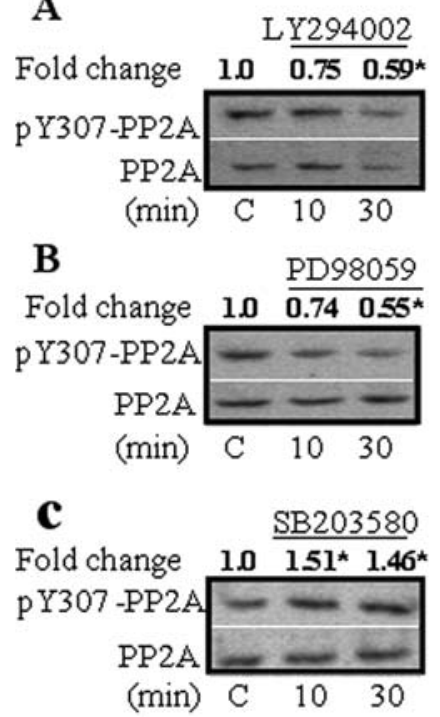

Figure 5. Effects of different inhibitors on tyrosine phosphorylation of PP2A. (A) Inhibition of PI3K specific inhibitor LY294002 on PP2A phosphorylation in BT474 cells. (B) Inhibition of MEK I inhibitor, PD98059, on the PP2A phosphorylation in BT474 cells. (C) Enhancement of p38 MAPK inhibitor, SB203580, on the PP2A phosphorylation in BT474 cells. BT474 cells at 70-80\% confluence were incubated with fresh medium containing inhibitor LY294002 $(10 \mu \mathrm{M})$, or PD98059 $(10 \mu \mathrm{M})$, or SB $203580(40 \mu \mathrm{M})$ for 10 and $30 \mathrm{~min}$. Cells were harvested and the level of PP2A and pY307-PP2A were detected by Western blotting. Relative tyrosine phosphorylation of PP2A was normalized to its basal level (pY307$\mathrm{PP} 2 \mathrm{~A} / \mathrm{PP} 2 \mathrm{~A}$ ), and interpreted as the fold change from triplicate experiments. ${ }^{*} \mathrm{p}<0.05,{ }^{* *} \mathrm{p}<0.01$ (Student's t-test).

intrinsic PI3K activity in BT474 cells was blocked with LY294002, a specific inhibitor of the p110 catalytic subunit of PI3K, the amount of pY307-PP2A formed was reduced. Similarly, a significant reduction of pY307-PP2A levels also occurred when the intrinsic MEK/ERK pathway was interrupted with the MEK I specific inhibitor, PD98059, which targets the MEK/ERK pathway (Fig. 5B). However, the cells treated with SB203580, a specific inhibitor of p38MAPK, showed an increased level of pY307-PP2A (Fig. 5C), implying that activation of p38MAPK reduces tyrosine phosphorylation of PP2A. This is consistent with the function of $\mathrm{p} 38$ as a tumour suppressor: activation of $\mathrm{p} 38 \alpha / \mathrm{B}$ causes cell growth arrest, senescence and/or apoptosis while inhibition of $\mathrm{p} 38 \alpha / \beta$ interrupts their growth arrest and resume proliferation $(20,21)$. The enhanced level of pY307-PP2A at the inhibitory state of p38MAPK could be attributed to inactivation of PP2A activity as a tumour suppressor, thereby facilitating cell survival. Taken together, our results clearly demonstrate that the tyrosine phosphorylation of PP2A in cells was regulated by a complex multi-signalling network, with PI3K/Akt and MEK/ERK exerting a positive effect, whereas p38MAPK has a negative effect on this essential process. Activation of PI3K or MEK could inactivate PP2A activity by enhancing its tyrosine phosphorylation. In contrast, activation of $\mathrm{p} 38 \mathrm{MAPK}$ could activate PP2A by inhibiting its tyrosine phosphorylation.

Tyrosine-phosphorylated PP2A was highly expressed in HER-2 positive breast tumours. We next investigated the expression of PP2A and PY307-PP2A in a panel of clinical
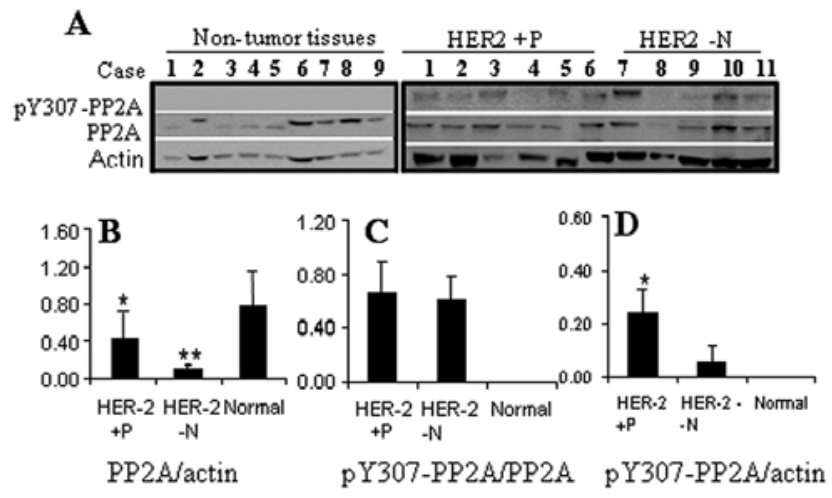

Figure 6. Expression of PP2A and tyrosine-phosphorylated PP2A (pY307PP2A) in clinical breast specimens. (A) Western blotting of PP2A and pY307-PP2A in tumour and non-tumour tissues. Total soluble proteins from both frozen breast tumour and non-tumour tissues were solubilized in Tissue Protein Extraction Reagent ${ }^{\circledR}$ (Pierce, Rockford, IL), after grounded to powder in liquid nitrogen. Proteins $(40 \mu \mathrm{g})$ were separated by SDS-PAGE (12\%), transferred to PVDF membranes, and immunodetected using antipY307-PP2A and anti-PP2A antibodies, followed by HRP-conjugated secondary antibody. Signals were captured with the MULTI GENIUS Bio Imaging System and signal intensity was analyzed by the GeneTools software. (B) Level of PP2A normalised to B-actin in breast tissues. (C) Relative phosphorylation of PP2A normalized to total PP2A. (D) Level of pY307-PP2A normalized to $B$-actin in breast tissues. Data represent the mean \pm SD of triplicate experiments. ${ }^{*} \mathrm{p}<0.05,{ }^{* *} \mathrm{p}<0.01$ (t-test).

specimens. As indicated in Fig. 6A, PP2A is expressed in both the tumour and non-tumour tissues. However, PP2A (normalized to $B$-actin) was highly expressed in the nontumour tissues and downregulated in the tumour tissues (Fig. 6B), further supporting the role of PP2A as a tumour suppressor in breast cancer progression. We also found that pY307-PP2A is only detected in the tumour tissues (Fig. 6A, right panel) and the level of pY307-PP2A (normalized to $B$-actin) is significantly increased in the HER-2 positive tumours, compared to the corresponding levels observed in the HER-2 negative tumours (Fig. 6D). However, the relative phosphorylation level of PP2A (pY307-PP2A/PP2A) was not markedly enhanced in the HER-2 positive tumours (Fig. 6C). Taking into account that phosphorylation of PP2A is a transient process under growth factor stimulation, as reported by Chen et al (18), and as demonstrated in our present study in an in vitro cell system, this transient phenomenon may not be readily observed in the tumour specimens.

To examine whether the level of pY307-PP2A is associated with the HER-2 status in breast cancer, we performed an immunohistochemical analysis with anti-pY307-PP2A antibody on tissue sections of a tissue microarray containing 35 cases of HER-2 positive tumours and 50 cases of HER-2 negative tumours, which we had constructed using a Beecher microarrayer (22). The extent/intensity of staining of pY307PP2A in both types of tumours was evaluated under light microscopy and scored as negative, moderate and strong staining. We showed that pY307-PP2A was positively stained (moderate/strong) in 15 of 25 HER-2 positive tumours and 14 of 44 HER-2 negative tumours ( $\mathrm{p}=0.04$, Fisher's exact test, data not shown). This result further demonstrated that inactivation of PP2A by tyrosine phosphorylation strongly correlated with the HER-2 status of the breast tumours. 


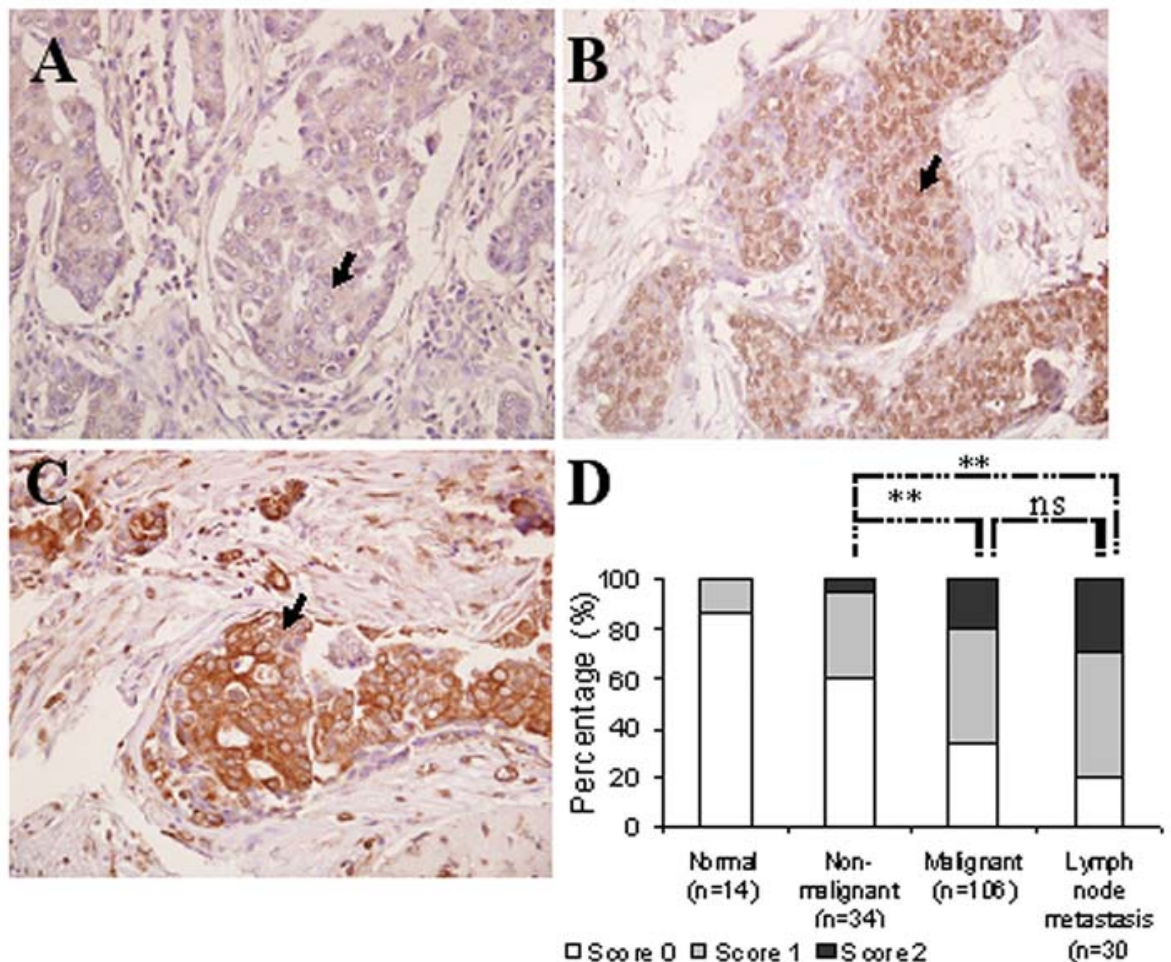

Figure 7. Representative immunohistochemical staining of pY307-PP2A on breast tissue microarrays. (A-C) showed staining level of score 0-2. Sections were deparaffinized in xylene and rehydrated in graded alcohols. After unmasking antigen and removing endogenous peroxidases, sections were incubated for $1 \mathrm{~h}$ with antibodies against pY307-PP2A at room temperature, followed by detection with labelled dextran polymer conjugated with peroxidase and $\mathrm{DAB}^{+}{ }_{-}$ substrate chromagen solution. Nuclei were lightly stained with Mayer's haematoxylin. The extent of staining was scored as 0 , 1 and 2 , according to the staining intensity of the tumour cells. (D) Distribution of pY307-PP2A expression in breast tissues. Breast tissues on the microarrays were classified as normal, non-malignant, malignant and lymph node metastasis. Data are expressed as percentage of cases with various scores in each subgroup. $<<0.05$ was considered as significant (Fisher's exact test). ${ }^{*} \mathrm{p}<0.05,{ }^{*} \mathrm{p}<0.01 ; \mathrm{ns}$, no significant difference. Arrows indicate the tumour cells.

Level of $p Y 307-P P 2 A$ correlated with breast cancer progression. To investigate whether $\mathrm{pY} 307-\mathrm{PP} 2 \mathrm{~A}$ expression is associated with breast cancer progression, we further analyzed the level of pY307-PP2A by immunohistochemistry on the tissue microarray we purchased from Biomax, which included 206 different cases. This array contained 32 cases of metastatic carcinomas, 116 malignant carcinomas, 44 nonmalignant tissues and 16 non-tumours. Scoring of the staining intensity of each case was made by two independent pathologists and one principal investigator. The staining intensity was classified as negative and weak (score 0 ), moderate (score 1) and strong (score 2) to indicate the expression of pY307-PP2A in different tissue specimens. In cases in which a discrepancy was noted, a subsequent review carried out to reach a consensus was mostly but not always successful; discordant results were excluded from statistical data analysis. Representative cases with different scores are shown in Fig. 7A, B and C). Of the 34 non-malignant tissues including benign growths, hyperplasia and inflammation, only 2 cases showed strong staining intensity and 12 moderate staining intensity, whereas in the 106 malignant carcinomas, 21 cases were strongly stained and 57 moderately stained $(\mathrm{p}<0.01)$. Noticeably, pY307-PP2A was highly stained in 8 of the 30 metastatic carcinomas, and with a higher frequency than that observed in the malignant carcinomas, although not at a significant level $(\mathrm{p}=0.15)$. None of the 14 normal tissues showed high expression of pY307-PP2A. Collectively, expression of pY307-PP2A was found to be progressively increased with the disease progression of the patients being assessed (Fig. 7D).

\section{Discussion}

Tyrosine phosphorylation is one of the central regulatory mechanisms of signal transduction in cancer progression. Proteins transiently or stably phosphorylated at tyrosine residues constitute the tyrosine phosphoproteome, a complex network leading to malignant transformation and disease. In breast cancer, HER-2 oncogene modulates cell survival and apoptosis by regulating its downstream signalling cascade of interlinked pathways, including those involving PI3K/Akt, Raf/MEK1/ERK and p38MAPK. To fully inventorize the tyrosine phosphoproteins involved in the HER-2 signalling network, phosphoproteomic profiles have been compiled using a variety of methods based on the SILAC, IMAC/ Nano-LC-MS/MS and antibody microarray platforms and novel tyrosine phosphoproteins have been identified $(6,7,23)$. These investigations were designed to support the concept that dissecting the changes in tyrosine phosphorylation of signal molecules would represent a fundamental task to elucidate the biologic/biochemical basis of disease progression and to develop therapeutic drugs targeting specific tyrosinephosphoproteins. Findings from some of these studies have provided the proof-of-concept sought. 
Table 1. Identified tyrosine-phosphorylated proteins from HRG-treated BT474 cells.

\begin{tabular}{|c|c|c|c|c|c|c|c|}
\hline Functional groups & Proteins & $\begin{array}{l}\text { Location } \\
\text { on arrays }\end{array}$ & $\begin{array}{c}\text { Tyrosine } \\
\text { phosphorylation } \\
\text { level }\end{array}$ & Functional groups & Proteins & $\begin{array}{l}\text { Location } \\
\text { on arrays }\end{array}$ & $\begin{array}{c}\text { Tyrosine } \\
\text { phosphorylation } \\
\text { level }\end{array}$ \\
\hline \multirow[t]{7}{*}{$\begin{array}{l}\text { Known HER2 } \\
\text { signaling proteins }\end{array}$} & erbB2 & L7 & Increased & $\begin{array}{l}\text { Cell cycle and } \\
\text { proliferation }\end{array}$ & CUL-1 & $\mathrm{T} 5$ & Increased \\
\hline & PI3K p85 & E15 & Increased & & Cyclin D3 & C6 & Increased \\
\hline & Akt1/2 & $\mathrm{F} 1$ & Increased & & GADD45 & $\mathrm{R} 8$ & Increased \\
\hline & SHC & L17 & Increased & & GADD153 & S8 & Increased \\
\hline & Stat1 & L18 & Increased & & P45 skp2 & G14 & Increased \\
\hline & Syk & B19 & Increased & $\begin{array}{l}\text { Signal transduction } \\
\text { related proteins }\end{array}$ & HCAM & K3 & Increased \\
\hline & Crk & R5 & Increased & & CD3 epsilon & K4 & Decreased \\
\hline \multirow{23}{*}{$\begin{array}{l}\text { Apoptosis and } \\
\text { anti-apoptosis } \\
\text { related proteins }\end{array}$} & BOK & D2 & Increased & & CD28 & M4 & Increased \\
\hline & $\mathrm{Bcl}-2$ & G2 & Increased & & Crk & R5 & Increased \\
\hline & BID & $\mathrm{N} 2$ & Increased & & EphA4 & $\mathrm{I} 7$ & Increased \\
\hline & $\mathrm{Bim}$ & $\mathrm{O} 2$ & Increased & & Eps8 & $\mathrm{K} 7$ & Increased \\
\hline & BAP1 & $\mathrm{R} 2$ & Increased & & p-EGFR & $\mathrm{C} 7$ & Decreased \\
\hline & Caspase3 & $\mathrm{R} 3$ & Increased & & Frizzled & P8 & Increased \\
\hline & Caspase4 & $\mathrm{S} 3$ & Increased & & HS1 & Q9 & Increased \\
\hline & CD40 & $\mathrm{O} 4$ & Increased & & IRAK & Q10 & Increased \\
\hline & FADD & $\mathrm{T} 7$ & Increased & & JNK1,2,3 & E11 & Increased \\
\hline & FLASH & K8 & Increased & & p-c-Raf-1 & E16 & Increased \\
\hline & FLIPs/1 & L8 & Increased & & Rap2 & $\mathrm{J} 16$ & Increased \\
\hline & GADD34 & Q8 & Increased & & $\mathrm{RXR}$ a,b,r & L16 & Increased \\
\hline & RAIDD & F16 & Increased & & TCR $\alpha$ & E19 & Increased \\
\hline & PAR-4 & $\mathrm{P} 14$ & Increased & & TCR $ß$ & F19 & Increased \\
\hline & SODD & S17 & Increased & & Patched & O14 & Increased \\
\hline & Apaf1 & M1 & Increased & & PDGF R & A15 & Increased \\
\hline & TNFR1 & M19 & Increased & & Rab1 & S15 & Increased \\
\hline & TNFR2 & N19 & Increased & & TANK & D19 & Increased \\
\hline & TRADD & P19 & Increased & & Throid R a1 & J19 & Increased \\
\hline & $\mathrm{ARC}$ & $\mathrm{O} 1$ & Increased & & & & \\
\hline & TRAF1 & Q19 & Increased & $\begin{array}{l}\text { Cell adhesion } \\
\text { related proteins }\end{array}$ & VCAM-1 & N3 & Increased \\
\hline & TRAF2 & R19 & Increased & & Bcl-6 & M2 & Increased \\
\hline & TRAIL & $\mathrm{C} 20$ & Increased & & Intergrin $\alpha 5$ & M10 & Increased \\
\hline \multirow[t]{13}{*}{$\begin{array}{l}\text { Transcription- } \\
\text { related proteins }\end{array}$} & ATF-2 & $\mathrm{P} 1$ & Increased & $\begin{array}{l}\text { Immune response } \\
\text { and cytokine } \\
\text { regulation }\end{array}$ & IL1R1 & D10 & Increased \\
\hline & Elongin A & G7 & Increased & & IRF2 & $\mathrm{S} 10$ & Increased \\
\hline & HDAC1 & L9 & Increased & & SOCS-1 & R17 & Increased \\
\hline & ICSBP & S9 & Increased & Tumor suppressor & Maspin & P11 & Increased \\
\hline & Id1 & T9 & Increased & & PP2A,2B & H15 & Increased \\
\hline & Max & Q11 & Increased & $\begin{array}{l}\text { DNA repair and drug } \\
\text { resistant proteins }\end{array}$ & $\operatorname{Rad} 52$ & $\mathrm{C} 16$ & Increased \\
\hline & MyoD & L12 & Increased & & Mdr & $\mathrm{T} 11$ & Increased \\
\hline & $\begin{array}{l}\text { NF-кB } \\
\text { p65 }\end{array}$ & $\mathrm{T} 12$ & Increased & $\begin{array}{l}\text { Cytoskeleton reorga- } \\
\text { nization proteins }\end{array}$ & & & \\
\hline & RACK1 & A16 & Increased & & Ezrin & S7 & Increased \\
\hline & Rar R & K16 & Increased & & B7-1 & $\mathrm{S} 1$ & Increased \\
\hline & c-Rel & T16 & Increased & & & & \\
\hline & Smad4 & Q17 & Increased & & & & \\
\hline & VDR & L20 & Increased & & & & \\
\hline
\end{tabular}

Relative tyrosine phosphorylation is expressed as the ratio of spot intensity on filter probed with the HRG-treated samples to that on the filter treated with control sample. Only the proteins with a ratio of $>1.5$-fold increased (or decreased) or only detected on the filter probed with HRG-treated samples (or on the filter probed with control sample) were considered as increased (or decreased) in their tyrosine phosphorylation. 
In this study, we investigated the HER-2-driven tyrosine phosphorylation profiles of the key signal modulators involved in various cellular functions using the signaling transduction antibody array (Hypromatrix), and identified 78 tyrosinephosphorylated proteins and 2 tyrosine-dephosphorylated proteins, tyrosine phosphorylation of which were stimulated or suppressed following HRG-enhanced HER-2 signalling. These differentially phosphorylated molecules are involved in diverse essential cell functions (apoptosis, transcription, cell adhesion, etc., as listed in Table I). In addition to specific downstream proteins (e.g., Shc, Stat1 and Akt) that were previously known to be upregulated by HER-2 signalling, we also found that most of the apoptosis-related proteins, e.g., FADD and ARC, were tyrosine phosphorylated when the BT474 cells were treated with HRG (Fig. 3), a first-time finding. Recently, Matsuyoshi et al found that FADD phosphorylation at Ser194 was regulated by the c-jun Nterminal kinase (JNK), affected cell cycle arrest and inhibited cell invasion (24), but whether tyrosine phosphorylation of FADD inactivated its apoptotic activity remains unclear. ARC is reported to be highly expressed primarily in brain and myogenic tissues (25). When cells are triggered by stimuli such as hydrogen peroxide, hypoxia and the Fas ligand, ARC appears to assume a protective role, inhibiting the cells from undergoing apoptosis $(26,27)$. However, little is known about the mechanism by which HER-2 signalling regulates tyrosine phophorylation of ARC in cancer cells. Mapping the tyrosine phosphorylation sites and elucidating its role in cancer cells would be critical to understanding the cross-talk between HER-2 signalling and cellular apoptotic signals. In addition, Ezrin, an important member of the ezrinradixin-moesin family involved in cell surface structures and cell survival $(28,29)$, was highly phosphorylated in the HRG-treated BT474 cells. Our finding was further supported by immunohistochemical study that phosphorylated ezrin was mostly found in HER-2/neu positive breast tumours (30).

Most intriguingly, we demonstrated that PP2A, a major protein serine/threonine phosphatase that participates in many signalling pathways in mammalian cells, was highly phosphorylated at tyrosine 307 in the HRG-treated BT474 cells (Fig. 4). PP2A modulates cell survival and apoptosis, and tyrosine phosphorylation of PP2A results in inactivation of its phosphatase activity and loss of function as a tumour suppressor. Despite full recognition of the importance of tyrosine phosphorylation in regulating its activity, the molecular mechanism of HER-2-triggered tyrosine phosphorylation of $\mathrm{PP} 2 \mathrm{~A}$ remains unknown. $\mathrm{PP} 2 \mathrm{~A}$ is a heterotrimer consisting of a $36-\mathrm{kDa}$ catalytic subunit (PP2A/C), a $65-\mathrm{kDa}$ structural A subunit (PP2A/A), and a variable regulatory subunit ranging in size from 50 to $130 \mathrm{kDa}$ (PP2A/B) (15). It has been found that phosphorylation at tyrosine 307 and/or methylation of the $\mathrm{C}$ subunit are the crucial steps in regulating PP2A activity $(18,31)$. Thus the posttranslational modification of PP2A is a critical regulatory step in exerting its role in mammalian cells, especially in cancer cells where it regulates cell survival and apoptosis by manipulating the activities of the key signal molecules, such as Akt, Bax, and Calpains (17,32-34). Given the importance of PP2A as a multifunctional modulator, molecular regulation of $\mathrm{PP} 2 \mathrm{~A}$ activity by the intrinsic and/or extrinsic signal network decides the cell's destination. Previous studies demonstrated that the Simian virus 40 small T antigen (SV40 ST), directly inhibits the activity of PP2A in promoting cell transformation (35). On the other hand, oncogenic signals enhanced by nonreceptor- and receptor-linked tyrosine kinases including the EGFR and the insulin receptor stimulate tyrosine phosphorylation of PP2A and thereby inhibit its activity (19). Considering the fact that EGFR and the related HER-2 are the most prevalent oncogenes in breast cancer, we propose that HER-2 signalling may also play a key role in 'tuning' PP2A activity by tyrosine phosphorylation as well. As expected, we found that tyrosine phosphorylation of PP2A was significantly affected either by switching on HER-2 signalling with HRG or by switching off HER-2 signalling with its specific inhibitor AG825 (Fig. 4). Our data further emphasize the importance of oncogenic signalling in regulating $\mathrm{PP} 2 \mathrm{~A}$ activity. Furthermore, the inhibitor experiments targeting PI3K, MEK1 and p38MAPK demonstrated their respective contribution to the tyrosine phosphorylation of PP2A. We found that tyrosine phosphorylation of PP2A was highly inhibited in BT474 cells by PI3K or MEK1 inhibitors, but was markedly increased in these same cells upon addition of the p38 MAPK inhibitor (Fig. 5). These results indicate that the PI3K/Akt and Raf/MEK1/Erk pathways positively regulate, whereas the p38MAPK pathway negatively regulates PP2A activity in cancer cells. These observations are consistent with the cell survival role of activated PI3K/Akt and Raf/MEK1/Erk and with the cell pro-apoptotic role of activated p38MAPK. Our data address and add credence to the concept that the key HER-2-linked downstream pathways constitute an interactive network to regulate the phosphorylation status of PP2A. This tyrosine phosphorylation-based modification of PP2A activity by the cross-signalling pathways in cancer cells further highlights its central role in regulating cell survival and apoptotic behaviour.

PP2A had been reported to be a tumour suppressor in breast, colon and lung tumours and myeloma (36). Seemingly in contradiction to the above study, PP2A was found to have a stimulatory effect in positively regulating Raf-1, a protooncogene upregulating the MAP kinase pathway (16), and in the Wnt-mediated signal transduction pathway (37). Furthermore, PP2A activity was shown to be required for cells to progress through the early phase of cell cycle to enter S phase (38), and to exert an anti-apoptotic effect on malignant testicular germ cell tumour (39). The contrasting functions of PP2A may be attributed to its existence as a multitasking, enzyme complex rather than as a single enzyme entity (14). Whilst it had been reported that PP2A activity was higher in ER-positive breast cancer cell lines than in the ER-negative cell lines (40), there is scanty information of pY307-PP2A expression in human breast tissues. To further investigate the distribution and expression of PP2A and pY307-PP2A in breast cancer specimens, and to understand whether the level of pY307-PP2A is associated with cancer progression, we tested the levels of both PP2A and pY307-PP2A in a panel of resected breast tumours. A comparison of the PP2A expression between tumour and non-tumour samples (Fig. 6B) further supported the role of PP2A as a tumour suppressor in breast cancer. Noticeably, the observation that pY307-PP2A was 
only detected in all the tumour samples, but not in any of the non-tumour samples (Fig. 6A and D), strongly suggests the inactivation of PP2A in tumours. Interestingly, the relative tyrosine phosphorylation of PP2A (pY307-PP2A/PP2A) was not significantly different in the HER-2-positive and -negative tumours (Fig. 6C). This is not surprising when one considers that as tyrosine phosphorylation induced by the oncogenic HER-2 signalling is a transient response, as verified by our data and supported by data from other studies using an in vitro cell system (18). This transient signal may not be readily observed in tumour samples. The increased expression of pY307-PP2A in the HER-2 positive tumours is consistent with the subsequent tyrosine phosphorylation and inactivation of PP2A expressed in this subtype of tumours. Moreover, tumours with higher positive staining of pY307-PP2A were associated with patients in the later stages of breast cancer progression, most notably in those with malignancy and lymph node metastasis (Fig. 7). This tissue microarray study supported the strong association of pY307-PP2A with tumour progression, and further highlighted the potential of pY307PP2A as a viable target therapy to block off tumorigenesis by its inherent property of reactivating PP2A activity. The results of our study also indicate that pY307-PP2A has a potential prognostic value in determining breast cancer progression.

In summary, our tyrosine phosphoproteomics study with the antibody array identified novel signal modulators that are highly phosphorylated and/or dephosphorylated under HER-2 signalling. These molecules constitute a group of potential targets in developing novel treatment regimes to arrest tumour progression in breast cancer cells by modulating their tyrosine phosphorylation status. The complicated regulatory mechanism of PP2A phosphorylation by the downstream pathways of HER-2 signalling reveals its essential role, at least in part, in deciding the destiny of these cells: the activated PI3K- and Raf/MEK pathways stimulate PP2A phosphorylation and attenuate its activity to facilitate cell survival, whereas the activated p38 MAPK pathway reduces PP2A phosphorylation and increases its activity to accelerate cell apoptotic activity. In the clinical tumour specimens, pY307-PP2A is highly increased in the more aggressive HER-2-positive breast tumours and markedly associated with tumour progression. Our investigation further indicates pY307-PP2A is an important prognostic factor in breast cancer development.

\section{Acknowledgements}

This work was supported by the Academic Research Grant from the Yong Loo Lin School of Medicine, National University of Singapore to E.S.K. and D.Z.

\section{References}

1. Reese DM and Slamon DJ: HER-2/neu signal transduction in human breast and ovarian cancer. Stem Cells 15: 1-8, 1997.

2. Holbro T, Civenni G and Hynes NE: The ErbB receptors and their role in cancer progression. Exp Cell Res 284: 99-110, 2003.

3. Slamon DJ, Clark GM, Wong SG, et al: Human breast cancer: correlation of relapse and survival with amplification of the HER-2/neu oncogene. Science 235: 177-182, 1987.

4. Spector NL, Xia W, Burris H, et al: Study of the biologic effects of lapatinib, a reversible inhibitor of ErbB1 and ErbB2 tyrosine kinases, on tumour growth and survival pathways in patients with advanced malignancies. J Clin Oncol 23: 2502-2512, 2005.
5. Grant S, Qiao L and Dent P: Roles of ERBB family receptor tyrosine kinases, and downstream signalling pathways, in the control of cell growth and survival. Front Biosci 7: D376-D389, 2002.

6. Mukherji M, Brill LM, Ficarro SB, Hampton GM and Schultz PG: A phosphoproteomic analysis of the ErbB2 receptor tyrosine kinase signalling pathways. Biochemistry 45: 15529-15540, 2006.

7. Bose R, Molina H, Patterson AS, et al: Phosphoproteomic analysis of HER2/neu signalling and inhibition. Proc Natl Acad Sci USA 103: 9773-9778, 2006.

8. Friedman DB, Wang SE, Whitwell CW, Caprioli RM and Arteaga CL: Multivariable difference gel electrophoresis and mass spectrometry: a case study on transforming growth factor-beta and ERBB2 signalling. Mol Cell Proteomics 6: 150-169, 2007.

9. Wolf-Yadlin A, Kumar N, Zhang Y, et al: Effects of HER2 overexpression on cell signalling networks governing proliferation and migration. Mol Syst Biol 2: 54, 2006.

10. Kumar N, Wolf-Yadlin A, White FM and Lauffenburger DA: Modeling HER 2 effects on cell behaviour from mass spectrometry phosphotyrosine data. PLoS Comput Biol 3: E4, 2007.

11. Zhang D, Tai LK, Wong LL, et al: Proteomic study reveals that proteins involved in metabolic and detoxification pathways are highly expressed in HER-2/neu-positive breast cancer. Mol Cell Proteomics 4: 1686-1696, 2005.

12. Wallasch C, Weiss FU, Niederfellner G, et al: Heregulindependent regulation of HER $2 /$ neu oncogenic signalling by heterodimerization with HER3. EMBO J 14: 4267-4275, 1995.

13. Dankort D, Jeyabalan N, Jones N, Dumont DJ and Muller WJ: Multiple ErbB-2/neu phosphorylation sites mediate transformation through distinct effector proteins. J Biol Chem 276: 38921-38928, 2001.

14. Janssens V and Goris J: Protein phosphatase 2A: a highly regulated family of serine/threonine phosphatases implicated in cell growth and signalling. Biochem J 353: 417-439, 2001

15. Schönthal AH: Role of serine/threonine protein phosphatase $2 \mathrm{~A}$ in cancer. Cancer Lett 170: 1-13, 2001.

16. Abraham D, Podar K, Pacher M, et al: Raf-1-associated protein phosphatase $2 \mathrm{~A}$ as a positive regulator of kinase activation. $\mathrm{J}$ Biol Chem 275: 22300-22304, 2000.

17. Andrabi S, Gjoerup OV, Kean JA, Roberts TM and Schaffhausen B: Protein phosphatase $2 \mathrm{~A}$ regulates life and death decisions via Akt in a context-dependent manner. Proc Natl Acad Sci USA 104: 19011-19016, 2007.

18. Chen J, Parsons S and Brautigan DL: Tyrosine phosphorylation of protein phosphatase $2 \mathrm{~A}$ in response to growth stimulation and v-src transformation of fibroblasts. J Biol Chem 269: 7957-7962, 1994.

19. Tsai CM, Levitzki A, Wu LH, et al: Enhancement of chemosensitivity by tyrphostin AG825 in high-p185(neu) expressing non-small cell lung cancer cells. Cancer Res 56: 1068-1074, 1996.

20. Bulavin DV and Fornace AJ: p38 MAP kinase's emerging role as a tumour suppressor. Adv Cancer Res 92: 95-118, 2004.

21. Aguirre-Ghiso JA, Estrada Y, Liu D and Ossowski L: ERK (MAPK) activity as a determinant of tumour growth and dormancy; regulation by p38(SAPK). Cancer Res 63: 1684-1695, 2003.

22. Zhang D, Tai LK, Wong LL, et al: Proteomic characterization of differentially expressed proteins in breast cancer: Expression of hnRNP H1, RKIP and GRP78 is strongly associated with HER-2/neu status. Proteomics Clin Appl 2: 99-107, 2008.

23. Gembitsky DS, Lawlor K, Jacovina A, Yaneva M and Tempst P: A prototype antibody microarray platform to monitor changes in protein tyrosine phosphorylation. Mol Cell Proteomics 3: 1102-1118, 2004.

24. Matsuyoshi S, Shimada K, Nakamura M, Ishida E and Konishi N: FADD phosphorylation is critical for cell cycle regulation in breast cancer cells. Br J Cancer 94: 532-539, 2006.

25. Koseki T, Inohara N, Chen S and Nunez G: ARC, an inhibitor of apoptosis expressed in skeletal muscle and heart that interacts selectively with caspases. Proc Natl Acad Sci USA 95: 5156-5160, 1998.

26. Ekhterae D, Lin Z, Lundberg MS, et al: ARC inhibits cytochrome c release from mitochondria and protects against hypoxia-induced apoptosis in heart-derived H9c2 cells. Circ Res 85: E70-E77, 1999

27. Hong YM, Jo DG, Lee JY, et al: Down-regulation of ARC contributes to vulnerability of hippocampal neurons to ischemia/ hypoxia. FEBS Lett 543: 170-173, 2003. 
28. Bretscher A: Regulation of cortical structure by the ezrinradixin-moesin protein family. Curr Opin Cell Biol 11: 109-116, 1999.

29. Gautreau A, Poullet P, Louvard D and Arpin M: Ezrin, a plasma membrane-microfilament linker, signals cell survival through the phosphatidylinositol 3-kinase/Akt pathway. Proc Natl Acad Sci USA 96: 7300-7305, 1999.

30. Sarrió D, Rodríguez-Pinilla SM, Dotor A, et al: Abnormal ezrin localization is associated with clinicopathological features in invasive breast carcinomas. Breast Cancer Res Treat 98: 71-79, 2006.

31. Favre B, Zolnierowicz S, Turowski P and Hemmings BA: The catalytic subunit of protein phosphatase $2 \mathrm{~A}$ is carboxylmethylated in vivo. J Biol Chem 269: 16311-16317, 1994.

32. Kong M, Fox CJ, Mu J, et al: The PP2A-associated protein alpha4 is an essential inhibitor of apoptosis. Science 306: 695-698, 2004

33. Grethe S and Pörn-Ares MI: p38 MAPK regulates phosphorylation of Bad via PP2A-dependent suppression of the MEK1/2ERK1/2 survival pathway in TNF-alpha induced endothelial apoptosis. Cell Signal 18: 531-540, 2006.

34. Xin $M$ and Deng X: Protein phosphatase 2A enhances the proapoptotic function of Bax through dephosphorylation. J Biol Chem 281: 18859-18867, 2006.
35. Sontag JM and Sontag E: Regulation of cell adhesion by PP2A and SV40 small tumor antigen: an important link to cell transformation. Cell Mol Life Sci 63: 2979-2991, 2006.

36. Janssens V, Goris J and Van Hoof C: PP2A: the expected tumour suppressor. Curr Opin Genet Dev 15: 34-41, 2005.

37. Ratcliffe MJ, Itoh K and Sokol SY: A positive role for the PP2A catalytic subunit in Wnt signal transduction. J Biol Chem 275: 35680-35683, 2000.

38. Yan Y and Mumby MC: Distinct roles for PP1 and PP2A in phosphorylation of the retinoblastoma protein. $\mathrm{PP} 2 \mathrm{~A}$ regulates the activities of $\mathrm{G}(1)$ cyclin-dependent kinases. J Biol Chem 274: 31917-31924, 1999

39. Schweyer S, Bachem A, Bremmer F, et al: Expression and function of protein phosphatase PP2A in malignant testicular germ cell tumours. J Pathol 213: 72-81, 2007.

40. Gopalakrishna R, Gundimeda U, Fontana JA and Clarke R: Differential distribution of protein phosphatase $2 \mathrm{~A}$ in human breast carcinoma cell lines and its relation to estrogen receptor status. Cancer Lett 136: 143-151, 1999. 